\title{
Osteoid Osteoma of the Intercondylar Notch: An Uncommon Cause of Knee Stiffness
}

\author{
Rachel M. Frank, MD ${ }^{1}$ Peter Nissen Chalmers, $\mathrm{MD}^{1} \quad$ Brian J. Cole, MD, MBA ${ }^{2} \quad$ Steven Gitelis, MD ${ }^{1}$ \\ ${ }^{1}$ Department of Orthopaedic Surgery, Rush University Medical Center, \\ Chicago, Illinois \\ 2 Department of Orthopedics, Division of Sports Medicine, \\ Rush University Medical Center, Chicago, Illinois Orthopaedic Surgery, Rush University Medical Center, 1611 West Harrison Street, Suite 300, Chicago, IL 60612 (e-mail: rmfrank3@gmail.com). \\ Address for correspondence Rachel M. Frank, MD, Department of
}

J Knee Surg Rep 2015;1:2-7.

\begin{abstract}
Keywords

- intercondylar notch

- osteoid osteoma

- stiffness

- arthroscopy

The presence of an osteoid osteoma in a periarticular or intra-articular location about the knee is rare. Osteoid osteoma of the knee may be present with nonspecific complaints including knee pain, stiffness, effusions, and atrophy. Depending on the clinical setting, these symptoms could represent a variety of different diagnoses, including meniscal pathology, chondral pathology, synovitis, or Plica syndrome. In this article, we present the unique case of an osteoid osteoma within the intercondylar notch of the knee that underwent a significant delay in diagnosis and several unnecessary procedures due to misdiagnosis. Although intra-articular osteoid osteomas have been described, there remains a paucity of case reports discussing these lesions within and around the knee, and currently there are no reports available describing such a lesion located within the intercondylar notch. Although osteoid osteomas are relatively common, the intra-articular location of such lesions presents a diagnostic challenge. Vague, nonspecific symptoms including joint pain, swelling, and mechanical symptoms coupled with nonspecific radiographic findings in an otherwise healthy, young patient are more likely to guide the clinician toward a more common diagnosis of a sportsrelated injury as opposed to a benign bone tumor. This case represents an extremely rare presentation of osteoid osteoma within the intercondylar notch of the knee. The case presentation provides an excellent reminder of the need to consider rare diagnoses such as osteoid osteoma, especially in cases of chronic, unrelenting, localized pain in the absence of any other explicable pathology.
\end{abstract}

Osteoid osteomas are self-limiting, benign, bone-forming neoplasms. ${ }^{1,2}$ First described by Jaffe in $1935,{ }^{3}$ these lesions are common and account for 10 to $12 \%$ of all benign bone tumors. These lesions almost always occur in patients younger than 30 years, are more common in males than females (approximately 2 to 1 ratio), and over $50 \%$ of the time are found within the metaphysis or diaphysis of long bones. Such lesions can be extremely painful, and the classic presentation involves a young patient with night pain in the tibia or femur that is relieved with oral salicylates. The presence of an osteoid osteoma in a periarticular or intra-articular location about the knee is rare. Osteoid osteoma of the knee may present with nonspecific complaints including knee pain, stiffness, effusions, and atrophy. Depending on the clinical setting, these symptoms could represent a variety of different diagnoses, including meniscal pathology, chondral pathology, synovitis, or Plica syndrome. Radiographic findings are often normal, and advanced imaging is required for diagnosis and treatment. Although intra-articular osteoid osteomas have been described, there remains a paucity of case reports received

January 31, 2013

accepted

April 14, 2013

published online

August 16, 2013
DOI http://dx.doi.org/

$10.1055 / \mathrm{s}-0033-1353419$. ISSN 2326-2729.
Copyright $\odot 2015$ by Thieme Medical

Publishers, Inc., 333 Seventh Avenue, New York, NY 10001, USA. Tel: +1(212) 584-4662.
License terms

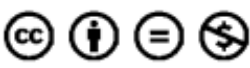


discussing these lesions within and around the knee, ${ }^{4-17}$ and currently there are no reports available describing such a lesion located within the intercondylar notch.

The purpose of this report is to present the unique case of an osteoid osteoma within the intercondylar notch of the knee that underwent a significant delay in diagnosis and several unnecessary procedures due to misdiagnosis. We also review the recent literature on the subject.

\section{Case Report}

The patient is a 24-year-old male, former collegiate football player, with a medical history of mitral valve prolapse, who presented to an outside physician with a chief complaint of right knee pain and limitation of range of motion without an inciting injury. On examination, he was noted to have anteromedial joint line tenderness and a significant loss of range of motion, although no specific measurements were given. No other examination findings were noted. He was provisionally diagnosed with a meniscal tear for which he underwent arthroscopic partial meniscectomy of the anteromedial horn.

Postoperatively, his pain and severe range of motion loss remained unchanged. Two months postoperatively, he returned to the operating room for insufflation brisement and manipulation under anesthesia. Despite aggressive physical therapy, his range of motion deficits persisted and he underwent an extensive arthroscopic lysis of adhesions followed by aggressive physical therapy. His limitation in range of motion was unchanged and thus he underwent an extensive capsular release including an arthroscopic-assisted mini-open posterior capsulotomy, again followed by persistent loss of range of motion. He then underwent a repeat insufflation brisement and manipulation under anesthesia. However, his stiffness remained unchanged. He was being considered for an open anterior and posterior complete synovectomy, circumferential capsulotomy, and lysis of adhesions when he presented to our office for a second opinion. At the time of presentation to our office, he had been in treatment for 2 years and had undergone a total of five surgical procedures.

At each of these prior procedures, the lateral meniscus and the remainder of the medial meniscus and anterior and posterior cruciate ligaments were noted to be intact. Synovitis of the intercondylar notch and chondromalacia of the inferior pole of the patella were noted and were debrided on several occasions.

At the time of presentation to our practice, the patient continued to experience diffuse right knee pain with associated stiffness in flexion and extension limiting his activity and hindering ambulation. He did not complain of effusions or mechanical symptoms. Since his last surgery, he had undergone a full course of physical therapy as well as several injections of corticosteroids, which had failed to improve his knee pain or stiffness.

Physical examination at the time of presentation revealed multiple well-healed incisions without evidence of infection. His range of motion was severely limited to 20 to 80 degrees of flexion. His knee was stable to ligamentous testing. He was diffusely tender in the knee without point tenderness referable to any specific anatomic structure. He was neurovascularly intact distally. Serologic testing revealed a normal white blood cell count, C-reactive protein level, and erythrocyte sedimentation rate.

Plain films obtained at the time of presentation as well as those reviewed from throughout his history demonstrated a round lucency at the proximal and medial aspect of the intercondylar notch visualized only on the partially flexed Rosenberg view. These radiographs were otherwise within normal limits (-Fig. 1). Review of a series of magnetic resonance imaging (MRI) studies obtained during the patient's course were reviewed, each demonstrating a $9 \times 10$ $\mathrm{mm}$-round osseous lesion at the junction of the anterior and medial walls of the intercondylar notch. The lesion was T2 hyperintense with a $2 \times 2-\mathrm{mm}$ central area of hypointensity and a rim of surrounding hypointensity. Extensive bony edema and intra-articular synovitis surrounded the lesion (-Fig. 2). Although postsurgical changes were seen elsewhere in the knee, no discrete pathology was visualized. Review of the patient's previous imaging demonstrated the lesion with interval changes only in the amount of surrounding edema and inflammatory signs ( $\mathbf{- F i g . 3}$ ). A bone scan was also obtained that demonstrated diffuse uptake to the right distal femur with a single spot of intense uptake at the proximal medial aspect of the notch (-Fig. 4).

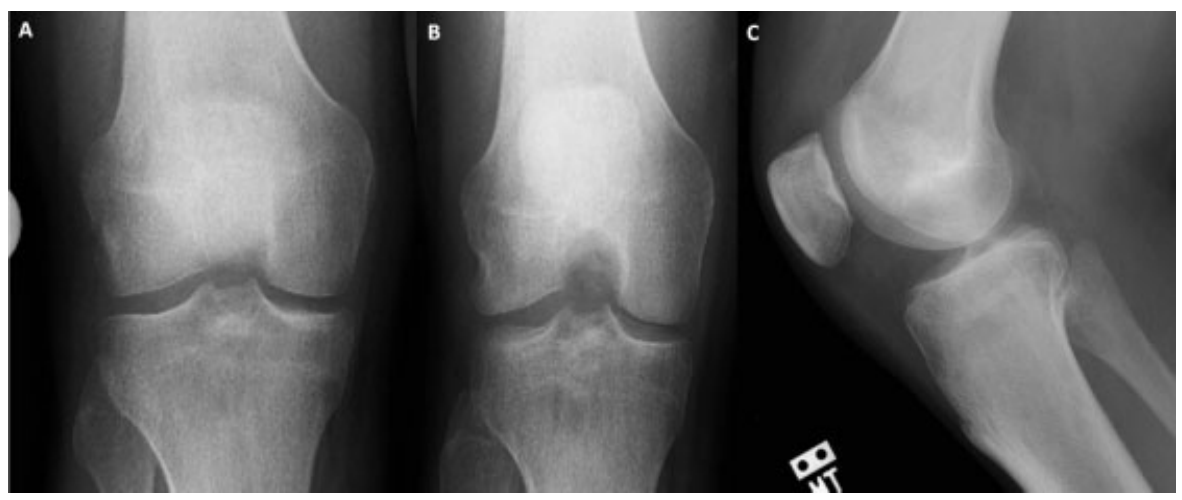

Fig. 1 (A) Anteroposterior, (B) 45-degree flexed posteroanterior (Rosenberg), and (C) lateral views of the right knee demonstrating round lucency at the proximal and medial aspect of the intercondylar notch visualized only on the partially flexed Rosenberg view. 


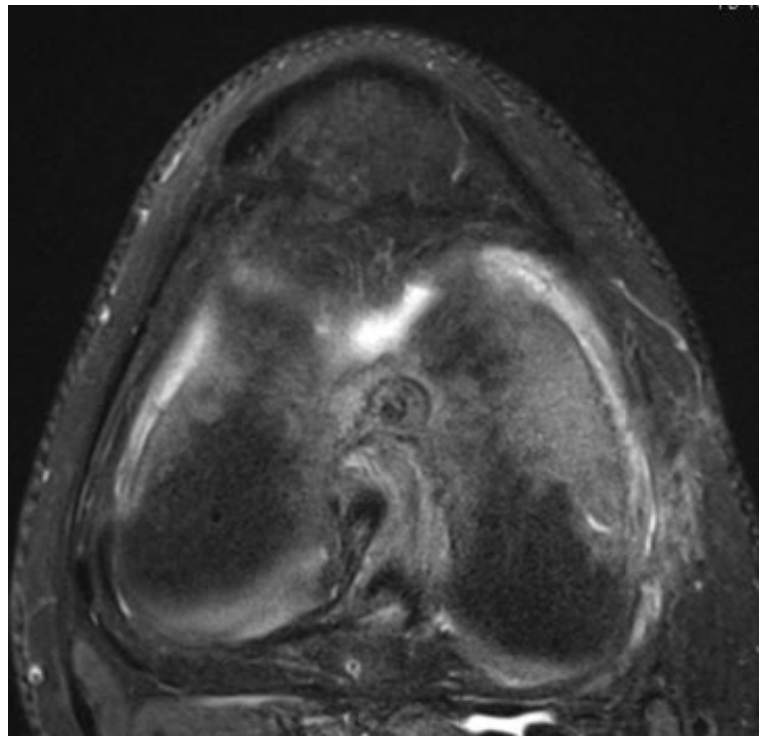

Fig. 2 Axial slice of a T2-weighted fat suppression magnetic resonance image at the level of the intercondylar notch of the femur demonstrating a $9 \times 10$-mm-round osseous lesion at the junction of the anterior and medial walls of the intercondylar notch with $\mathrm{T} 2$ hyperintensity, a $2 \times 2-\mathrm{mm}$ central area of hypointensity, and a rim of surrounding hypointensity. Extensive bony edema and intra-articular synovitis surrounds the lesion.

Based upon these findings the patient was given a provisional differential diagnosis of chondroblastoma, osteoid osteoma, and osteomyelitis. Given the concern for chondroblastoma and the high likelihood of lesional spillage with an arthroscopic procedure, he underwent a limited open arthrotomy with excisional biopsy. Intraoperatively, the lesion was identified as an outpouching of the articular cartilage of the notch, with a soft-tissue filled cavity of the

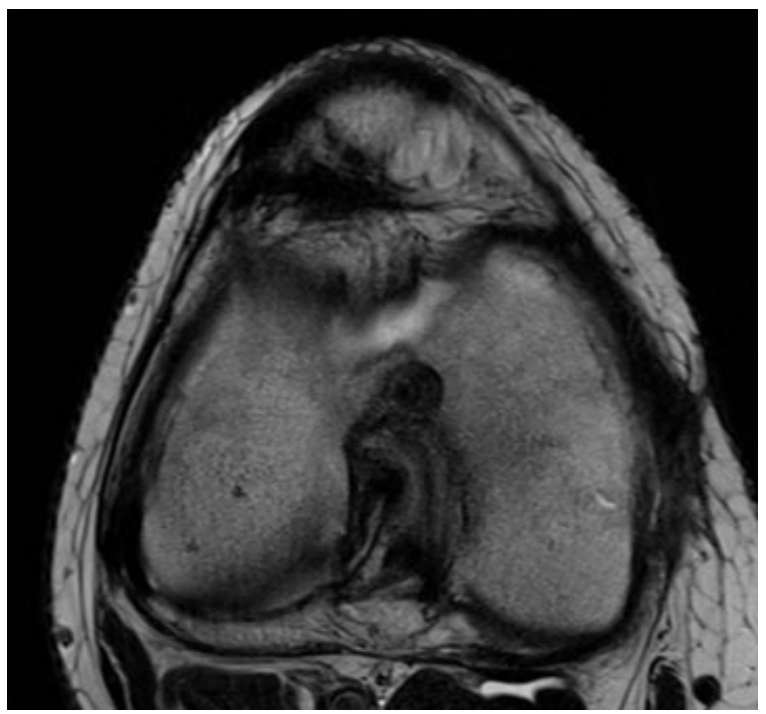

Fig. 3 Axial slice of a T1-weighted magnetic resonance image at the level of the intercondylar notch of the femur from one prior to Fig. 2 redemonstrating an identical lesion.

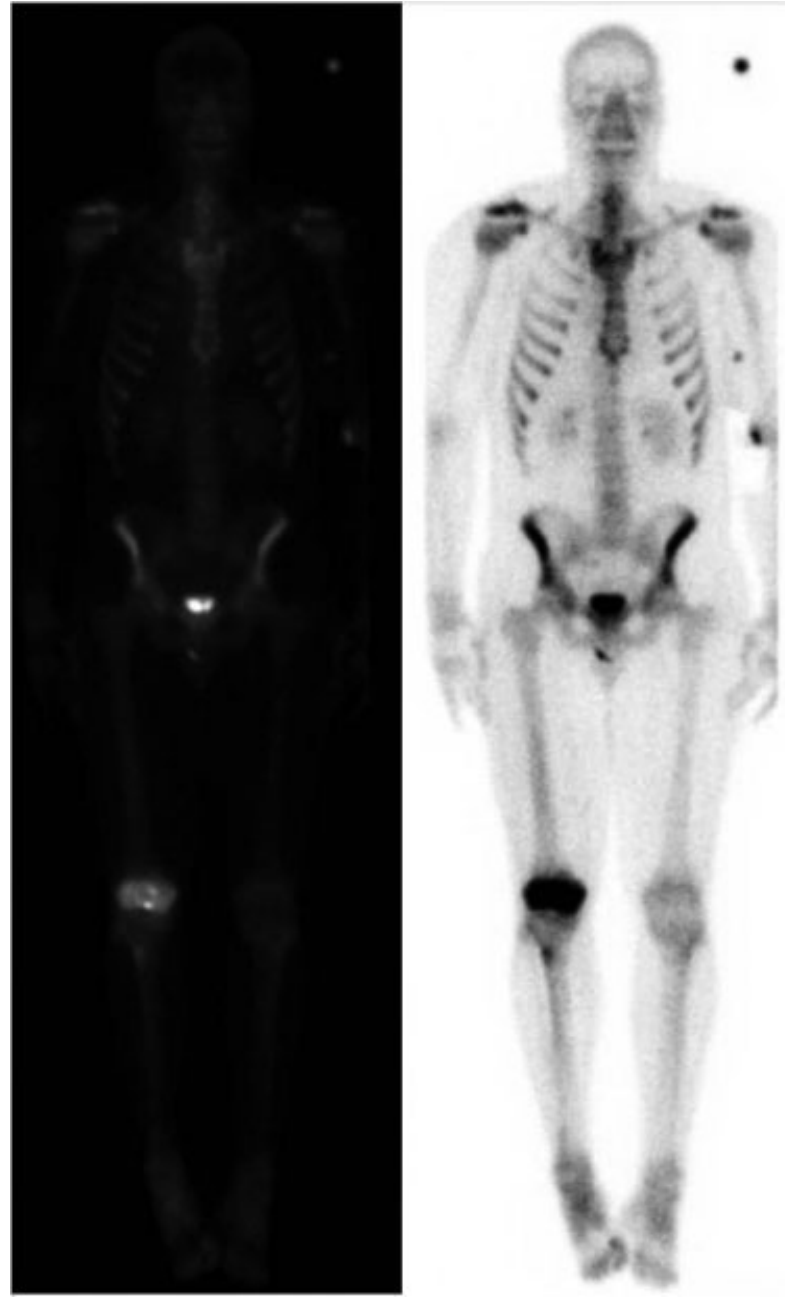

Fig. 4 Full-body image from the delayed phase of a Technitium-99 bone scan demonstrating diffuse uptake to the right distal femur with a single spot of intense uptake at the proximal medial aspect of the notch.

subchondral bone beneath, the contents of which were sent for biopsy and culture. The lesion was positioned such that the cartilage removed was nonarticular and did not involve the attachment of either of the cruciate ligaments. The lesion subsequently underwent curettage, burring, and cauterization. Closure was performed in the usual fashion. Final pathology was consistent with osteoid osteoma (- Fig. 5). All cultures were negative.

Upon initial follow-up 2 weeks postoperatively, the patient's preoperative knee pain had completely resolved. The patient was prescribed physical therapy to aggressively work on range of motion. The patient was initially planned for a second arthroscopic procedure with lysis of adhesions in a staged fashion. However, by 4 weeks postoperatively, the patient had recovered full painless range of motion from 0 to 120 degrees of flexion and thus second procedure was unnecessary. This arc of motion exceeded any he had obtained since before orthopedic evaluation 2 years preoperatively. At final follow-up 1 year postoperatively, the patient had recovered full range of motion from 0 to 130 degrees and 


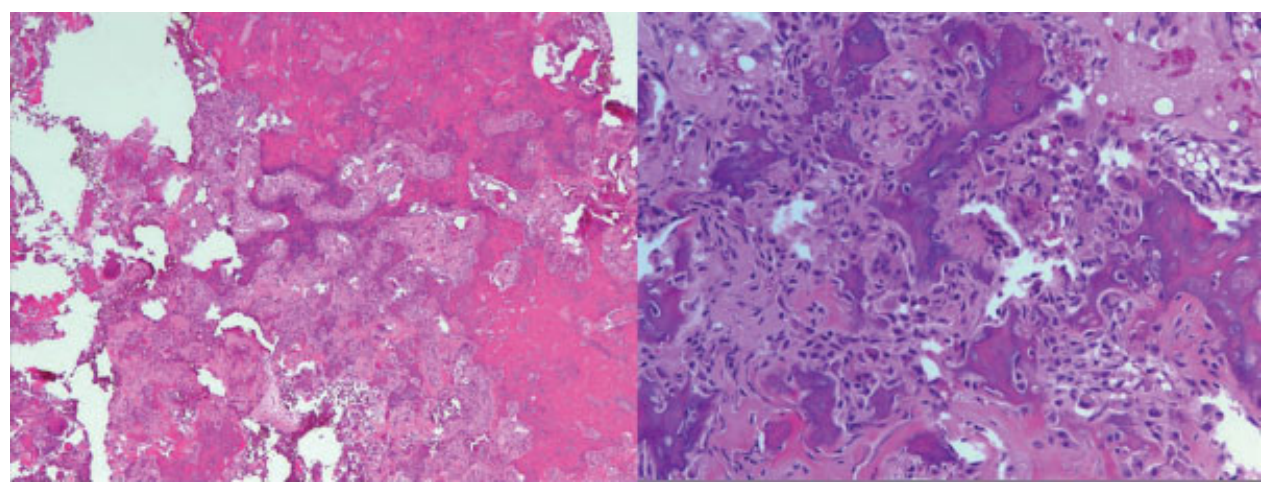

Fig. 5 Low- and high-power histologic representation of intraoperative biopsy demonstrating findings consistent with osteoid osteoma. Note the mixture of immature osteoid with osteoblastic rimming and reactive bone seen in the sclerotic border.

had no residual knee pain. He had returned to his full activities including high-impact athletics without discomfort.

\section{Discussion}

This case represents a rare presentation of an osteoid osteoma in the knee of an otherwise healthy, young, and athletic patient. Osteoid osteomas are self-limiting bone-forming neoplasms. Histologically, they are characterized by a small core, or nidus, composed of woven bone and osteoid, lined with osteoblasts and intermixed with highly vascularized connective tissue. ${ }^{1,2}$ There is a clear area at the periphery of the nidus because of osteoclastic bone resorption occurring simultaneously with osteoblast-driven remodeling. The nidus is surrounded by a distinctive zone of reactive, dense, sclerotic bone. This is often visible on plain radiographs with lesions along the metaphysis or diaphysis of long bones. The nidus in osteoid osteoma is less than 1.5 to $2 \mathrm{~cm}$ in its greatest dimension. Larger lesions are referred to as osteoblastomas, which are histologically similar but are locally more aggressive and also less responsive to salicylates when compared with osteoid osteomas.

The majority of osteoid osteomas occur along the metaphyseal or diaphyseal cortex of long bones, most often in the femur or tibia. ${ }^{1,2}$ Other common locations include the spine, upper extremity, pelvis, hands, and feet. Clinically, patients with osteoid osteomas typically present with localized pain at the site of lesion; the pain is worse at night and often responds to nonsteroidal anti-inflammatories (NSAIDs). ${ }^{1,2}$ Several theories exist to explain the painful nature of osteoid osteomas. Of note, nerve fibers are present adjacent to the arterioles within the nidus, and may likely be responsible for the painful nature of these lesions. ${ }^{18}$ Another likely pain generator is abundant prostaglandin synthesis within the nidus, which may explain the effectiveness of NSAIDs. ${ }^{19}$

When the lesion is located near a joint, the patient may present with an effusion, synovitis, early degenerative changes, stiffness, and possibly even joint contractures. Given these symptoms, juxta-articular osteoid osteomas may mimic other, more common diagnoses, especially in young athletic patients in which sports-related injuries are much more common. ${ }^{14,16,20-23}$ Furthermore, juxta-articular osteoid osteomas may not have the classic radiographic findings characteristically found with metaphyseal and diaphyseal lesions. This may be related to a lack of peripheral sclerosis surrounding the nidus of osteoid osteomas near articulations. $^{24}$ Computed tomography (CT) is better equipped to differentiate between the nidus and surrounding trabecular bone. ${ }^{21}$

Clearly, such nonspecific signs and symptoms combined with inconclusive radiographic findings may ultimately result in a delayed diagnosis, as was demonstrated in our case. As was noted by Abnousi et al, ${ }^{9}$ juxta-articular osteoid osteomas may result in significant delays in diagnosis (months to years) leading to inadequate treatment. As such, the clinician must consider this diagnosis in cases of persistent, otherwise unexplained, monoarticular pain in young, otherwise healthy patients. ${ }^{14,16,20-24}$ Also of importance is the consideration of recalcitrant stiffness as seen in our case, which is unusual even for osteoid osteomas.

Despite the relatively high prevalence of these benign neoplasms, the literature is replete with case reports of rare presentations of osteoid osteoma. Various reports of osteoid osteoma in the hand, ${ }^{25-31}$ foot, $^{13,32-37}$ shoulder, ${ }^{12,20,38}$ and even the face, ${ }^{39}$ and teeth ${ }^{40}$ are available. As previously mentioned, although osteoid osteomas are common, they most often occur in long bones, the spine, and pelvis, and thus these isolated case reports do indeed represent rare occurrences. Despite the anatomical differences between these cases, one uniting feature of nearly all of these reports is continued, unrelenting, localized pain associated with a delay in appropriate diagnosis. The authors of these reports commonly conclude that the diagnosis of osteoid osteoma must be considered in cases of young, otherwise healthy patients with chronic, unexplained, localized pain.

With regard to the knee, several scattered reports that analyze rare presentations of osteoid osteoma within the proximal tibia, distal femur, and even the patella ${ }^{4-17}$ are available. In 1985, Torg et al ${ }^{16}$ reported on 11 cases of osteoid osteoma in physically active people with a mean duration of symptoms of approximately 20 months before appropriate diagnosis. The majority of these patients complained of referred pain to the knee, and were found to have lesions either distant from the knee, periarticular lesions, or subarticular 
(intra-articular) lesions. The authors noted less than half of the cohort had characteristic radiographic findings, and the remaining patients (6 of 11) required advanced imaging including bone scans, tomograms, and CT scans before the appropriate diagnosis was made. Our current case is unique in that the presentation was most unusually associated with recalcitrant stiffness, whereas in other cases pain was the primary symptom. Specifically, our patient presented with range of motion from 20 to 80 degrees, and his main concern was continued stiffness leading to pain. Other reports ${ }^{9}$ have emphasized pain above any other presenting symptom.

On the basis of the classification system discussed by Torg et al, ${ }^{16}$ our case represents an intra-articular osteoid osteoma. As seen intraoperatively, the lesion was visualized as an outpouching of the articular cartilage within the notch, with a soft-tissue filled cavity of subchondral bone beneath. The treatment options for osteoid osteomas are variable, ${ }^{1,2,8,10,12,13,41-43}$ and nonoperative management with NSAIDs is the logical first step. In the current case, the patient had clearly already failed multiple efforts of both prior nonoperative and operative management; however, it should be noted that the prior operative attempts did not address the actual lesion. Surgical management for osteoid osteomas of the long bones and periarticular region is appropriate when pain persists to the point where it becomes problematic even with activities of daily living. Surgical options include open excision, CT-guided percutaneous excision, and CT-guided radiofrequency ablation. In the present case, given the nondiagnostic imaging findings and nonspecific presentation, we had some concern for chondroblastoma and thus elected to perform a limited open arthrotomy with excisional biopsy. The obvious risks with this treatment choice include damage to the articular cartilage, menisci, and nearby ligament attachments; however, in this case, the lesion was positioned such that the cartilage removed was nonarticular and did not involve the attachment of either of the cruciate ligaments.

Overall, although osteoid osteomas are relatively common, the intra-articular location of such lesions is rare and presents a diagnostic challenge. Vague, nonspecific symptoms including joint pain, swelling, and mechanical symptoms coupled with nonspecific radiographic findings in an otherwise healthy, young patient are more likely to guide the clinician toward a more common diagnosis of a sports-related injury as opposed to a benign bone tumor. This case represents an extremely rare presentation of osteoid osteoma within the intercondylar notch of the knee. The case presentation provides an excellent reminder of the need to consider rare diagnoses such as osteoid osteoma, especially in cases of chronic, unrelenting, localized pain in the absence of any other explicable pathology.

\section{References}

1 Atesok KI, Alman BA, Schemitsch EH, Peyser A, Mankin H. Osteoid osteoma and osteoblastoma. J Am Acad Orthop Surg 2011; 19(11):678-689

2 Gitelis S, Schajowicz F. Osteoid osteoma and osteoblastoma. Orthop Clin North Am 1989;20(3):313-325
3 Jaffe HL. Osteoid osteoma: a benign osteoblastic tumor composed of osteoid and atypical bone. Arch Surg 1935;31:709-728

4 Ma K, Zhao HT, Niu XH, Zhang Q. Osteoid osteoma of the patella: report of two cases. Chin Med J (Engl) 2011;124(23):4096-4098

5 Furukawa M, Anazawa U, Horiuchi K, et al. Arthroscopic removal of intra-articular osteoid osteoma in the knee: case report and review of the literature. J Orthop Sci 2011;16(3):321-325

6 García-Germán D, Sánchez-Gutiérrez S, Bueno A, et al. Intraarticular osteoid osteoma simulating a painful fabella syndrome. Knee 2010;17(4):310-312

7 Chaabane S, Merghani A, Drissi C, Ladeb MF. Knee pain in a 17year-old girl. Clin Orthop Relat Res 2010;468(7):2003-2006

8 Gunes T, Erdem M, Bostan B, Sen C, Sahin SA. Arthroscopic excision of the osteoid osteoma at the distal femur. Knee Surg Sports Traumatol Arthrosc 2008;16(1):90-93

9 Abnousi F, Saliman JD, Fanton GS. Arthroscopic visualization and assisted excision of osteoid osteoma at the knee: a case report and review. Am J Sports Med 2008;36(2):375-378

10 Eggel Y, Theumann N, Lüthi F. Intra-articular osteoid osteoma of the knee: clinical and therapeutical particularities. Joint Bone Spine 2007;74(4):379-381

11 Vallianatos PG, Tilentzoglou AC, Seitaridis SV, Mahera HJ. Osteoid osteoma of the patella: a case report. Knee Surg Sports Traumatol Arthrosc 2006;14(2):161-164

12 Franceschi F, Marinozzi A, Papalia R, Longo UG, Gualdi G, Denaro E. Intra- and juxta-articular osteoid osteoma: a diagnostic challenge : misdiagnosis and successful treatment: a report of four cases. Arch Orthop Trauma Surg 2006;126(10):660-667

13 Mastrokalos DS, Pässler HH, Tibesku CO, Wrazidlo W. Computed tomography-guided endoscopic removal of an osteoid osteoma from the femur. Arthroscopy 2001;17(1):62-66

14 Safran MR, Fu FH. Uncommon causes of knee pain in the athlete. Orthop Clin North Am 1995;26(3):547-559

15 Heuijerjans W, Dandy DJ, Harris D. Arthroscopic excision of an intra-articular osteoid osteoma at the knee. Arthroscopy 1986; 2(4):215-216

16 Torg JS, Loughran T, Pavlov H, et al. Osteoid osteoma. Distant, periarticular, and subarticular lesions as a cause of knee pain. Sports Med 1985;2(4):296-304

17 Matera D, Campanacci DA, Caldora P, Mazza E, Capanna R. Osteoid osteoma of the femur with a double nidus: a case report. Chir Organi Mov 2005;90(1):75-79

18 Schulman L, Dorfman HD. Nerve fibers in osteoid osteoma. J Bone Joint Surg Am 1970;52(7):1351-1356

19 Makley JT, Dunn MJ. Prostaglandin synthesis by osteoid osteoma. Lancet 1982;2(8288):42

20 Georgoulis AD, Soucacos PN, Beris AE, Xenakis TA. Osteoid osteoma in the differential diagnosis of persistent joint pain. Knee Surg Sports Traumatol Arthrosc 1995;3(2):125-128

21 Szendroi M, Köllo K, Antal I, Lakatos J, Szoke G. Intraarticular osteoid osteoma: clinical features, imaging results, and comparison with extraarticular localization. J Rheumatol 2004;31(5): 957-964

22 Muscolo DL, Ayerza MA, Makino A, Costa-Paz M, Aponte-Tinao LA. Tumors about the knee misdiagnosed as athletic injuries. J Bone Joint Surg Am 2003;85-A(7):1209-1214

23 Norman A, Abdelwahab IF, Buyon J, Matzkin E. Osteoid osteoma of the hip stimulating an early onset of osteoarthritis. Radiology 1986;158(2):417-420

24 Sim FH, Dahlin CD, Beabout JW. Osteoid-osteoma: diagnostic problems. J Bone Joint Surg Am 1975;57(2):154-159

25 Tonogai I, Hamada Y, Yasui N. A case of osteoid osteoma of the trapezoid bone: the efficiency of dynamic magnetic resonance imaging for the detection of osteoid osteoma localized at the atypical site. Hand Surg 2012;17(1):99-103

26 Rubin G, Wolovelsky A, Rinott M, Rozen N. Osteoid osteoma of the hamate: an unusual cause of ulnar-sided wrist pain. Orthopedics 2010;33(7):513 
27 Galdi B, Capo JT, Nourbakhsh A, Patterson F. Osteoid osteoma of the thumb: a case report. Hand (NY) 2010;5(4):423-426

28 Bostan B, Sen C, Gunes T, Erdem M, Koseoglu RD. Osteoid osteoma of the trapezium: case report. J Hand Surg Am 2010;35(4): 636-638

29 Arora J, McLauchlan J, Munro N. Recurrent osteoid osteoma of the lunate: a case report and review of the literature. Hand Surg 2003;8(2):239-242

30 Chamberlain BC, Mosher JF, Levinsohn EM, Greenberg JA. Subperiosteal osteoid osteoma of the hamate: a case report. J Hand Surg Am 1992;17(3):462-465

31 Tsang DS, Wu DY. Osteoid osteoma of phalangeal bone. J Formos Med Assoc 2008;107(7):582-586

32 Aratake M, Shigeyuki M, Atsushi H, Takeuchi R, Saito T. Case of juxta-articular osteoid osteoma of calcaneus mimicking arthritis. J Foot Ankle Surg 2012;51(2):237-240

33 Gürkan V, Orhun H, Bülbül M, Kayahan S. Osteoid osteoma of the cuboid bone: a rare cause of foot pain. Acta Orthop Traumatol Turc 2011;45(1):66-69

34 Mavrogenis AF, Dimitriou R, Benetos IS, Korres DS, Papagelopoulos PJ. Juxta-articular osteoid osteoma of the talar neck: a case report. Clin Podiatr Med Surg 2010;27(4):629-634

35 Sproule JA, Khan F, Fogarty EE. Osteoid osteoma: painful enlargement of the second toe. Arch Orthop Trauma Surg 2004;124(5): 354-356
36 Lakkis S, Bazzi JS, Shabb NS. Osteoid osteoma of the proximal phalanx of a toe. A case report. Bull Hosp Jt Dis 1998;57(2): 102-104

37 Gao K, Hua Y, Chen S, Li Y, Xin Q. Arthroscopic excision of juxtaarticular cancellous osteoid osteoma in the talar neck. Knee Surg Sports Traumatol Arthrosc 2013;21(6):1300-1303

38 Poyanli O, Unay K, Akan K, Ozkan K, Temiz D. Subchondral osteoid osteoma of the glenoid. Chir Organi Mov 2009;93(Suppl 1): S79-S81

39 Strong EB, Tate JR, Borys D. Osteoid osteoma of the ethmoid sinus: a rare diagnosis. Ear Nose Throat J 2012;91(5):E19-E20

40 Mohammed I, Jannan NA, Elrmali A. Osteoid osteoma associated with the teeth: unusual presentation. Int J Oral Maxillofac Surg 2013;42(2):298-302

41 Lindner NJ, Ozaki T, Roedl R, Gosheger G, Winkelmann W, Wörtler K. Percutaneous radiofrequency ablation in osteoid osteoma. J Bone Joint Surg Br 2001;83(3):391-396

42 Muscolo DL, Velan O, Pineda Acero G, Ayerza MA, Calabrese ME, Santini Araujo E. Osteoid osteoma of the hip. Percutaneous resection guided by computed tomography. Clin Orthop Relat Res 1995; (310):170-175

43 Zoccali C, Teori G, Salducca N, Di Paola B, Adriani E. Arthroscopic guided biopsy and radiofrequency thermoablation of a benign neoplasm of the tibial spines area: a treatment option. BMC Musculoskelet Disord 2012;13:52 